\title{
Trayectorias institucionales, diagnósticos psiquiátricos y violencias combinadas en un estudio de caso
}

\author{
Silvia Guemureman, Ph.D. \\ Universidad de Buenos Aires, Argentina*
}

Eugenia Bianchi, Ph.D.

Universidad de Buenos Aires, Argentina **

silviaguemureman@gmail.com

\section{Resumen (analítico)}

Se abordan las categorías de trayectorias institucionales, diagnósticos psiquiátricos y violencias, a través de un estudio de caso de un adolescente declarado penalmente responsable y luego condenado por la comisión de delitos en Ciudad de Buenos Aires (1993-2005), a través de su expediente tutelar abierto en los juzgados de menores, desde que era menor no punible hasta su mayoría de edad. El análisis se complementa con las sentencias definitivas y normativa relacionada. El objetivo es analizar este expediente desde el concepto de trayectorias, hipotetizando que estas no son lineales, deterministas ni reduccionistas, sino que se componen en una densa trama de circunstancias y experiencias. Los resultados se organizan en tres vectores: diagnósticos psiquiátricos, trayectorias institucionales y violencias. El artículo busca contribuir teórica y metodológicamente a los estudios sociales de juventudes.

\section{Palabras clave}

Instituciones, juventud, violencia, trayectorias, derecho penal.

\section{Thesauro}

Lista de descriptores Clacso.

\section{Para citar este artículo}

Guemureman, S., \& Bianchi, E. (2020). Trayectorias institucionales, diagnósticos psiquiátricos y violencias combinadas en un estudio de caso. Revista Latinoamericana de Ciencias Sociales, Niñez y Juventud, 18(3), 1-26.

https://dx.doi.org/10.11600/1692715x.18307

\section{Historial}

Recibido: 23.10 .2020

Aceptado: 10.02 .2020

Publicado: 26.08 .2020

\section{Información artículo}

Este artículo forma parte de los resultados de nuestro trabajo como investigadoras a tiempo completo del Consejo Nacional de Investigaciones Científicas y Técnicas (Conicet) de Argentina, y se inscribe en nuestros actuales proyectos de investigación en curso, a saber: «Escenarios de reforma legislativa en materia de responsabilidad penal adolescente y administración de justicia» (S. Guemureman) y «Fármacos, diagnósticos y saberes profesionales: estudio sobre las transformaciones en salud mental infantil en Argentina (1994-2020)» (E. Bianchi). Se siguieron lineamientos de investigación cualitativa. Área: Sociología. Temas especiales: estudios de infancia y adolescencia; ciencias sociales; agencias de control sociopenal, salud mental. 


\section{Institutional trajectories, psychiatric diagnoses and violence in a case study}

\section{Abstract (analytical)}

The categories of institutional trajectories, psychiatric diagnoses and violence are addressed through a case study of an adolescent declared criminally responsible and later convicted of ordering crimes in Buenos Aires (1993-2005). His institutional trajectories were followed through analysis of his custody file in the juvenile courts as he was a minor not punishable until he reached the age of criminal responsibility. This analysis is complemented with the inclusion of the adolescent's final sentences, and related regulations. The objective of the case study is to analyze this file using the concept of trajectories, hypothesizing that these are not linear, deterministic or reductionist, but consist of a dense fabric of circumstances and experiences. The results are organized into three vectors: psychiatric diagnoses, institutional trajectories and violence. The article seeks to contribute theoretically and methodologically to the field of youth studies.

Keywords

Institutions, youth, violence, trajectories, criminal Justice.

\section{Trajetórias institucionais, diagnósticos psiquiátricos e violências combinadas em um estudo de caso}

\section{Resumo (analítico)}

As categorias de trajetórias institucionais, diagnósticos psiquiátricos e violências são abordadas, através de um estudo de caso de um adolescente declarado criminalmente responsável e depois condenado pela prática de crimes na cidade de Buenos Aires (1993-2005), através de seu arquivo tutelar aberto nos julgados juvenis, uma vez que era menor de idade não punível até a maioridade. A análise é complementada pelas sentenças finais e regulamentos relacionados. O objetivo é analisar esse arquivo a partir do conceito de trajetórias, levantando a hipótese de que não são lineares, determinísticos ou reducionistas, mas que são compostos por um conjunto denso de circunstâncias e experiências. Os resultados estão organizados em três vetores: diagnóstico psiquiátrico, trajetórias institucionais e violência. $\bigcirc$ artigo busca contribuir teórica e metodologicamente para os estudos sociais da juventude.

\section{Palavras-chave}

Instituições, juventude, violência, trajetória, direito Penal.

\section{Información autoras}

[*] Universidad de Buenos Aires (UBA), Argentina. Consejo Nacional de Investigaciones Científicas y Técnicas (Conicet), Argentina. Consejo Latinoamericano de Ciencias Sociales (Clacso). Licenciada en Sociología. Especialista en Problemáticas Sociales Infanto-Juveniles. Doctora en Ciencias Sociales (UBA). Docente de grado y posgrado. iD 0000-0002-0187-0409. Índice H5: 0. Correo electrónico: silviaguemureman@gmail.com

[**] Universidad de Buenos Aires (UBA), Argentina. Consejo Nacional de Investigaciones Científicas y Técnicas (Conicet), Argentina. Consejo Latinoamericano de Ciencias Sociales (Clacso). Licenciada en Sociología. Magíster en Investigación en Ciencias Sociales. Doctora en Ciencias Sociales (UBA). Docente de grado y posgrado. iD 0000-0003-2311-7490. Índice H5: 2. Correo electrónico: eugenia.bianchi@gmail.com 


\section{Introducción}

$\mathrm{E}_{\text {penalmente responsable y posteriormente condenado por la comisión de distin- }}^{1}$ tos delitos en Ciudad de Buenos Aires, entre 1993 y 2005. El análisis de realiza a través del expediente tutelar, es decir, el legajo que se abre en la sede de administración de justicia, en el cual se registran todas las intervenciones realizadas sobre la persona y que dan cuenta de su respuesta a las medidas implementadas como tratamiento tutelar luego de declarada la disposición a cargo del juzgado. Este expediente no contiene las actuaciones penales, que se llevan por separado en otro expediente (el expediente actuario). Esta descripción expresa el tipo de documento que integra nuestro corpus, entre otros: informes de equipos técnicos diversos, delegados tutelares, informes de instituciones o programas a los que el sujeto en cuestión haya sido derivado.

El objetivo es analizar este expediente a partir del concepto de trayectorias, hipotetizando que estas no son lineales, deterministas o reduccionistas, sino que, por el contrario, se componen y devienen en una densa y compleja trama de diferentes circunstancias y experiencias. En ellas se articulan los diagnósticos psiquiátricos, la peligrosidad y el riesgo, el tránsito por diferentes espacios institucionales jurídicos y psiquiátricos, las violencias y la caída en el sistema penal. El análisis se complementa con el texto de las sentencias definitivas (segundas sentencias) dictadas sobre aquel al que denominaremos Luis.

Los resultados provienen de una sistematización analítica basada en fuentes documentales de una investigación de posgrado, articuladas con perspectivas teórico-metodológicas no exploradas previamente, y que arrojaron conclusiones noveles. Se consideran tres conceptos de diferentes líneas de estudios sociales que se articulan en función del análisis del caso: trayectorias institucionales, diagnósticos psiquiátricos y violencias en la doble dimensión de padecimiento y ejercicio.

Los resultados se organizan en tres vectores. El primero aborda la trayectoria de Luis en relación a los diagnósticos - mayormente psiquiátricos-que fue recibiendo y que 
sustentaron intervenciones institucionales avaladas por la instancia judicial. El segundo se enfoca en las trayectorias en relación a las instituciones que transitó Luis en el período consignado en el expediente. El tercer vector describe la trayectoria de violencias asociadas (padecidas y ejercidas) crecientes en intensidad, en la que Luis estuvo incluido.

El artículo procura ofrecer aportes en diferentes planos que presentamos separadamente a fines expositivos. Por una parte, en términos teóricos, busca contribuir a los estudios de trayectorias sociales, niñez y juventud; en particular sobre aquellas juventudes cuyas acciones concitan el reproche penal. Los estudios de trayectorias vienen siendo empleados en infancias, adolescencias y juventudes para analizar instituciones de inclusión, socialización primaria y formación para la vida ciudadana (familia, escuela, trabajo). Nuestro aporte es el empleo de la matriz de estudios de trayectorias para dar cuenta de los recorridos de niños, niñas, adolescentes y jóvenes que frecuentan el sistema penal y el rutero institucional que pivotea entre instituciones de seguridad y de índole psiquiátrica y terapéutica. El artículo entrama estos estudios con conceptualizaciones que vinculan el padecimiento mental con la peligrosidad, el riesgo y la violencia.

Los aportes se continúan en la apuesta metodológica, las cual combina el estudio de caso y el análisis de documentos jurídicos (el expediente tutelar y la segunda sentencia). Ambos recursos metodológicos se integran en una estrategia cualitativa de análisis de fuentes, a la que se añade el estudio de normativa relacionada.

\section{Trayectorias y juventudes en la trama conceptual}

El concepto de trayectorias es central para analizar el expediente tutelar. Seguimos los desarrollos de Macri y Uhart (2017), quienes remiten la relevancia de este concepto a estudios anglosajones y europeos, destacando que esos aportes fueron revisados en estudios latinoamericanos que abordan las trayectorias de adolescentes y jóvenes en diversas dimensiones de la vida social. En su escrito, rastrean las nociones de trayectoria escolar, carrera escolar y biografía escolar, relacionados a su vez con el concepto de habitus, en tanto este vertebra la explicación de las conductas asociadas a la trayectoria escolar (Bourdieu \& Passerón, 1996). Con este concepto, los autores aluden a la línea de vida, carrera o camino, de dirección, grado y proporción (variables a lo largo de la vida), por lo que resulta relevante para analizar las trayectorias de los jóvenes.

Para analizar sujetos que, como Luis, exhiben un historial de prácticas desviadas y llevan a cabo acciones que se leen a la luz de la configuración de una identidad desviada, la sociología de la desviación ha apelado al concepto de carrera (Becker, 1974) para dar 
cuenta de lo que sucede a estos jóvenes en los procesos de criminalización primaria y secundaria, y cómo activan procesos de desviación (Lemert, 1967; Matza, 1981). Estos temas se inscriben en los estudios de subculturas criminales (Cloward \& Ohlin, 20oo; Cohen, 1955). La noción de carrera está asociada al estudio de dinámicas más lineales que no permiten — como sí lo hace la noción de trayectoria - captar clivajes, ambigüedades, marchas, contramarchas e imbricaciones entre saberes, poderes e instituciones como las documentadas en el caso de Luis. Por ello, sostenemos que el concepto de trayectoria supera el de carrera, dada su capacidad de tomar la historia en su conjunto e integrarla.

También nos interesa retener que Macri y Uhart diferencian el concepto de transición — de cuño estadounidense- de la noción de trayectoria. Aplicada a la juventud, la transición es representada (como marcan Dávila-León y Ghiardo-Soto, 2005) como un período intermedio, a la vez pasaje y espera entre dos estados, sea infancia y adultez o antes y después. Los autores aclaran que entender a la juventud como transición no significa que esta sea una etapa de pura latencia, inercia o moratoria.

El concepto de trayectoria permite una aproximación no lineal a las historias de vida de adolescentes y jóvenes. Esto habilita la consideración de temporalidades no acotadas a un principio y fin específicos (como el año de ingreso y egreso al sistema escolar), sino de más larga duración, en las que el tránsito no describe una recta ni está preestablecido en relación a modelos societales y puede caracterizarse antes bien por la desestructuración, diversificación y espiralamiento y, por lo tanto, por las múltiples transiciones. Como también marcan las autoras, diversos estudios trabajan la dimensión temporal desde enfoques acerca de los procesos vitales de los actores sociales, los cuales destacan la relación entre estructura y agencia, así como su pertinencia para analizar niñas, niños y jóvenes (Macri \& Uhart, 2017).

En Argentina se están debatiendo y sistematizando aportes de estudios de trayectorias en relación específicamente a jóvenes (Chávez, 2010; Chávez \& Fidalgo-Zeballos, 2013; Kossoy, 2012; Rovacio et al., 2016). En esa línea, Torillo (2011) reseña y discute diferentes corrientes que intersectan los estudios sobre jóvenes y trayectorias, aportando una consideración útil para reflexionar en relación al corpus que analizamos aquí. Puntualmente, este autor señala que

metodológicamente el concepto de trayectoria implica cambiar la mirada del investigador desde la óptica de los escenarios que remiten a una visión cristalizada, estática; por el con- 
trario, el concepto de trayectoria implica atender a la dinámica, a la temporalidad y a la movilidad. (p. 21)

A esta clave nos atenemos para analizar el caso de Luis, y ello se detalla la propuesta metodológica que presentamos a continuación.

\section{Método}

La elección teórica del concepto de trayectoria como eje del análisis se continúa en el diseño metodológico. Los estudios de trayectoria admiten diseños tanto cualitativos como cuantitativos, pero en ambos exigen considerar la dimensión temporal a fin de captar procesos vitales o ciclos de vida de los actores sociales, de forma tal que se aporte a la comprensión de la relación entre agencia y estructura social (Macri \& Uhart, 2017). Entre las metodologías cualitativas, el enfoque de trayectorias sociales ocupa un lugar destacado en investigaciones orientadas al conocimiento de matices y alternativas biográficas de las personas en diferentes dimensiones de su vida, así como en relación a sus condiciones estructurales a lo largo del tiempo (Chávez, 2010). Ello resulta adecuado para captar vínculos entre diagnósticos psiquiátricos, tránsito por espacios institucionales y violencias en jóvenes que cometen delitos y son capturados por el sistema penal.

También en sintonía con la noción de trayectorias, el estudio de caso se presenta como una estrategia para fundamentar una aproximación no cristalizada ni estática, sino dinámica y con eje en la temporalidad y la movilidad, con foco en el seguimiento de las personas. Como también plantea Archenti (2007), la diversidad de tipologías acerca de los estudios de caso expresa la importancia de caracterizar a los mismos «tanto en sus criterios definitorios como en los objetivos que motivan su aplicación, a fin de esclarecer su alcance y relevancia» (p. 245).

Sin desestimar los aportes de diferentes autores que han elaborado tipologías y señalado las limitaciones, debates y aportes de esta estrategia (Archenti, 2007; Neiman \& Quaranta, 2006), dados los objetivos de este artículo nos concentramos en algunas tipologías para una caracterización general del caso de Luis. Así, y aunque la clasificación no es excluyente, el análisis del corpus de documentos sobre Luis puede considerarse como lo que Stake (1994) denominó estudio de caso instrumental, porque oficia de mediación para comprender un fenómeno más amplio. Aunque en un sentido negativo (que es resaltado por los jueces que tienen a su cargo el expediente de Luis), su caso también puede 
definirse - desde la tipología de Stake-como ejemplar. Siguiendo la tipología de Yin (2014) se trata de un estudio de caso único y puede entenderse simultáneamente como un caso infrecuente y revelador. Desde el análisis de Mallimaci y Giménez-Béliveau (2006), el estudio de caso se vincula con las historias de vida y los métodos biográficos. Puntualmente, «la historia de vida es el estudio de un individuo o familia y su experiencia de largo plazo (...) surgida de un trabajo con documentos y otros registros vitales» (p. 176), tomándolos como sujetos activos con capacidad de tomar decisiones que afectan sus trayectorias.

Para Flyvbjerg (2006) los estudios de caso se prestan a confusiones, entre ellas la idea de que el conocimiento derivado de ellos no permite arribar a conclusiones más generales. En concreto, para este autor, el que no se pueda generalizar formalmente

no significa que no pueda tomar parte en el proceso colectivo de la acumulación del conocimiento en un campo determinado o en una sociedad dada. Un estudio de caso fenomenológico puramente descriptivo sin ningún intento de generalizar puede, sin duda, tener algún valor en este proceso y con frecuencia ha contribuido a abrir un camino hacia la innovación científica (...). Simplemente pretendo hacer hincapié en las limitaciones que se producen cuando la generalización formal se convierte en el único método legítimo de investigación científica. (p. 42)

En consonancia con lo expuesto por Flyvbjerg, y desde el planteamiento de Ferrarotti $(1988,1991)$ en relación a la representatividad de las muestras, las historias de vida, no solo proporcionan información sobre un sujeto individual, sino que buscan expresar problemáticas y temas de la sociedad o un sector de esta. Por ello, para Ferrarotti la representatividad no es objetivo de esta estrategia.

Por lo expuesto, los estudios de caso constituyen una estrategia o diseño de investigación (antes que un método en sí mismo) que, por lo general, se vincula con métodos de análisis cualitativos (Archenti, 2007), y que se definen más por el interés en un objeto de estudio que por una elección metodológica determinada (Stake, 1994). Tal objeto puede abordarse desde diferentes metodologías y con distintas técnicas de recolección y análisis de datos, siempre que resulten adecuadas para el logro de un análisis intenso y profundo de la unicidad de los significados; lo anterior con la premisa de comprender el caso en su especificidad y no de establecer regularidades o inferencias generalizadoras. Entre tales técnicas, se cuenta el análisis de documentos (Archenti, 2007). 
Sin adentrarnos en tales técnicas, sí nos interesa puntualizar que los expedientes y sentencias son especies de textos judiciales específicos que cementan su estructura en la fundamentación de las decisiones de quienes las enuncian (sea el juez, el fiscal, el asesor, el defensor o el equipo técnico) y también en la configuración de lógicas discursivas que utilizan diferentes estrategias lingüisticas. Ello en pos de reforzar, jerarquizar o mitigar argumentaciones de acuerdo con una estructura formal (Cucatto, 2009; Pardo, 1992; Parodi, 2008).

El discurso de expedientes y sentencias es jurídico y con distintos atributos; entre ellos, el carácter argumentativo, lógico y retórico que lo define, a la vez que se sirve de múltiples mecanismos y dispositivos teóricos que lo dotan de una modalidad singular de construcción o invención de la realidad jurídica (Bourdieu, 200o; Cucatto, 2009; Mari, 2002; Martínez-García, 1992; Pardo, 1992). Esta singularidad permite abordarlos con metodologías cualitativas, tanto desde el análisis lingüístico (Agüero \& Zambrano, 2009; Vasilachis de Gialdino, 1992), como desde el análisis crítico del discurso (Van Dijk, 2003) y el análisis en calidad de género discursivo (Agüero, 2014; Bajtin, 1982; Parodi, 2008) o macrogénero discursivo (Parodi, 2008, 2009).

\section{Aspectos éticos}

El expediente fue relevado en un Tribunal Oral de Menores de la Ciudad de Buenos Aires. Se acordó confidencialidad en el tratamiento de datos sensibles y anonimización de referencias que pudieran conducir a la identificación, en consonancia con la Resolución 2857/2006 del Comité de Ética del Consejo Nacional de Investigaciones Científicas y Técnicas (Argentina, 2006). Se modificaron nombres propios incluidos en el expediente y se conservaron datos de trayectorias institucionales y recorridos de Luis, omitiendo nombres de instituciones, directivos y profesionales.

\section{El caso Luis}

Luis había nacido en Chile en 1982. El primer ingreso al Servicio Social de la Dirección Social del Menor se había producido en 1993, cuando contaba con 11 años, bajo la figura de vagancia, vigente en el código contravencional y de faltas de entonces. Para entonces, el padre de Luis había regresado a Chile y formado otra familia. La madre permaneció en Argentina con varios hijos, profundizando una situación de severa vulnerabilidad. Luis y su familia en Argentina habitaban un asentamiento informal en la Ciudad de Buenos 
Aires. Deambulando por andenes de los ferrocarriles, inició el consumo de pegamento y pedía dinero a los transeúntes.

En este contexto se produce la primera aprehensión, que deriva en el ingreso a un instituto de menores. A esta le seguirán otras, motivadas primero por contravenciones (vagancia, ebriedad y otras intoxicaciones) y luego por delitos (robo simple y luego robo agravado). En las primeras vueltas del ciclo su madre lo va a buscar. Desde la tercera vez, él mismo se fuga. Refuerza su irreductibilidad con silencios, negativas a reconocer los hechos, a justificarlos o cualquier otra enunciación que prometiese un cambio sustantivo de conducta y que pudiera ser tomada para disminuir el reproche penal (Guemureman, 2011). Los escasos registros de sus palabras que constan en el expediente, consignan enunciados con consideraciones tales como que es un placer robar y que no dejará de hacerlo.

\section{Un mínimo encuadre normativo}

El análisis del expediente de Luis también requiere considerar aspectos que remiten al encuadre normativo general vigente en Argentina en el período analizado. Puntualmente, tomamos el Decreto Ley 22278 sobre Régimen Penal de la Minoridad, sancionado por la Comisión de Asesoramiento Legislativo en plena dictadura cívico-militar (Argentina, 1980). Desde su sanción generó controversias, dado que deposita en el juez una amplia potestad para resolver las causas y lo dota de plenas facultades sobre los menores, sean estos infractores a la ley, imputables o no. De este modo, puede dictaminar medidas restrictivas, tales como la privación de libertad o el alojamiento en instituciones específicas de tratamiento, e inclusive prolongar tales medidas hasta que el sujeto arribe a la mayoría de edad. Diversas posturas doctrinarias criticaron la atribución de estas facultades omniabarcativas, y la ley en su conjunto fue fuertemente cuestionada (Beloff, 2004; Beloff \& García-Méndez, 2004; García-Méndez \& Bianchi, 1991; García-Méndez \& Carranza, 1992; Guemureman, 2011).

El centro de la polémica es el artículo $4^{\circ}$, conocido como perdón judicial; es decir, la facultad soberana del juez de proceder en forma indulgente, perdonando en virtud de atender a consideraciones de madurez (esto es, atendiendo a la persona y no al acto). Este artículo permite comprender cómo se castiga a los y las jóvenes: la efectiva imposición de una pena a un adolescente autor de delitos únicamente puede ser aplicada si el o la joven ya cumplió los 18 años, si además estuvo sometido(a) a tratamiento tutelar al menos por un año y si, previo a esto, se le había declarado la responsabilidad penal; es 
decir, si el tribunal había evaluado técnicamente que el o la adolescente había sido efectivamente autor o coautor del delito de referencia.

Una vez cumplidos estos requisitos, si las modalidades del hecho, los antecedentes del menor, el resultado del tratamiento tutelar y la impresión directa recogida por el juez hicieren necesario aplicarle una sanción, así lo resolverá, pudiendo reducirla en la forma prevista para la tentativa. Contrariamente, si fuese innecesario aplicarle sanción, lo absolverá, en cuyo caso podrá prescindir del requisito que el autor o coautor del delito hubiese cumplido dieciocho años.

La declaración de responsabilidad penal no supone de por sí la imposición de una pena luego de transcurrido el año de tratamiento tutelar, sino que constituye al decir de los jueces un juicio exclusivamente técnico (Guemureman, 2011) en que se valoran hecho y prueba. Para su dictado no se consideran los informes obrantes en el expediente tutelar que sigue la evolución de los y las jóvenes.

Luego de trascurrido un año desde que el adolescente fuese dispuesto tutelarmente, el tribunal está en condiciones de resolver definitivamente la causa, a partir de los resultados del tratamiento tutelar. Este último exige un período de observación que no puede ser inferior al año. Durante su transcurso, el adolescente puede estar sometido a distintas medidas restrictivas, como la privación de libertad en alguna institución específica (instituto de menores, comunidad terapéutica, etc.), el seguimiento y control por medio de un operador de libertad asistida, la sujeción a visitas domiciliarias de delegados tutelares o la obligación de comparecer al tribunal en determinados períodos, para el control y seguimiento in situ.

Todas estas medidas, a su vez, pueden complementarse con tratamientos médicos, psicológicos o psiquiátricos, recomendación de capacitaciones y realización de estudios o cursos y, eventualmente, constancias de ejecución de trabajos rentados. En definitiva, se trata de conocer cómo el adolescente hace uso de su tiempo libre: si en forma productiva o improductiva, y con arreglo a proyectos vitales constructivos o destructivos.

\section{Acerca del expediente tutelar}

El expediente se abre según lo dispuesto por el Decreto Ley 22278 (Argentina, 1980) con el objetivo de consignar los pasos dados por Luis, desde que fuera dispuesto tutelarmente. El expediente tutelar se abre en la primera instancia en el juzgado de menores, y acompaña al sujeto menor de edad a lo largo de la tramitación de su/s causa/s. Es 
frecuente que hechos distintos motiven la apertura de causas distintas y la consecuente apertura de expedientes tutelares diferentes. No obstante, según la normativa que regula la competencia de los tribunales, un solo tribunal está facultado a proseguir las actuaciones. Es frecuente que los expedientes tutelares expresen una intensa comunicación entre secretarios actuarios y tutelares de los distintos juzgados, que se remiten las respectivas actuaciones. La apertura de expedientes diferentes da lugar a la superposición de información una vez acumulados estos, en los cuales debe corregirse la foliatura. Casos paradigmáticos los configuran aquellos adolescentes que han activado la instancia judicial en múltiples ocasiones.

El expediente tutelar de Luis estuvo abierto más de 10 años (desde 1993 hasta 2005) y cuando se accedió al mismo las actuaciones ya estaban archivadas; es decir, que al compromiso de confidencialidad y la anonimización de la identidad del sujeto, se añade el beneficio de archivo. La relevancia de este expediente reside en que fue abierto con anterioridad a que el adolescente fuera punible y se fue engrosando con actuaciones hasta que llegó a su mayoría de edad (que en el período analizado se alcanzaba a los 21 años).

Con respecto a las características materiales del expediente, el mismo consta de seis cuerpos. Reconstruir su contenido implicó 15 horas de registro en audio de sus diferentes secciones, luego desgrabadas y copiadas en procesador de textos. El desorden del expediente tutelar y la superposición de actuaciones de varios juzgados tornaron imposible una reconstrucción lineal.

Los diferentes cuerpos del expediente almacenaban cantidad de oficios librados interjuzgados, los cuales habían sido derivados al expediente tutelar al apercibirse que el otro juzgado ya tenía uno en trámite. Luis había pasado prácticamente por todos los juzgados de la ciudad. Y aunque entre mediados de 1993 y 1995 no consta ninguna información, no puede desestimarse la posibilidad de que durante ese período Luis se haya encontrado a disposición de tribunales de otras jurisdicciones (como la Provincia de Buenos Aires) o institucionalizado.

\section{La segunda sentencia o sentencia del $4^{\circ}$}

El proceso penal de personas menores de edad implica el dictado de una primera y una segunda sentencia. La primera sentencia juzga el hecho y la prueba, concluyendo con la declaración de responsabilidad penal —en caso de corresponder- o el sobreseimiento. En esta instancia, el fiscal hace su pretensión de pena, la cual deja en suspenso a expensas de la evaluación del año de tratamiento tutelar. Transcurrido ese lapso, debe pronun- 
ciarse la segunda sentencia o sentencia definitiva, también conocida como sentencia del $4^{\underline{0}}$, anclada en el artículo $4^{\underline{0}}$ del Decreto Ley 22278 (Argentina, 1980).

Al momento de pronunciar la segunda sentencia, se consideran los resultados del tratamiento tutelar, a los que se asigna una carga valorativa que lo califica como positivo o negativo. Lo que se evalúa combina una ideología correccional y terapéutica, con pretensiones de protección y pedagogía. Para su seguimiento se examinan aspectos de la vida de los menores con parámetros valorados como positivos socialmente: escuela, familia, actitud respecto a las normas y el trabajo. Estos aspectos son tamizados por el sutil filtro de la moral de cada juez. Dependiendo su criterio y estimación sobre los resultados favorables o desfavorables del tratamiento tutelar, pueden absolver, reducir pena o imponer la pena prevista de acuerdo al delito imputado (Guemureman, 2011).

La segunda sentencia de Luis hacía referencia a diferentes declaraciones de responsabilidad penal relacionadas con hechos atribuidos al sujeto, que se iniciaron cuando ya este era punible, es decir, entre sus 16 y 17 años. Las acciones delictivas producidas a partir de los 18 años - y que concitaron reproche penal- suscitaron la intervención de diferentes juzgados penales ordinarios. Para cuando se pronunció la segunda sentencia, el Tribunal de menores interviniente realizó el cómputo de la sentencia de unificación. A estas particularidades se añade que se trata de un caso emblemático que interseca en la vulneración extrema de derechos y el padecimiento psíquico (Guemureman, 2018).

\section{Resultados}

Presentamos a continuación los resultados del análisis, que organizamos en tres vectores convergentes. Como postulamos en la hipótesis, en cada vector se delinean caminos sinuosos, no lineales, elípticos o cíclicos. El primer vector aborda la trayectoria de Luis en relación a los diagnósticos, mayormente psiquiátricos, que fue recibiendo y que acompañaron y sustentaron las actuaciones judiciales. El segundo se enfoca en las trayectorias en relación a las instituciones que transitó Luis en el período consignado en el expediente. Mientras que el tercer y último vector describe la trayectoria de violencias padecidas y ejercidas en la que Luis estuvo incluido. Aunque a efectos analíticos estos tres vectores se presentan por separado, en el expediente no siempre es posible seccionar cada temática. 


\section{Trayectorias sinuosas: la hibridez entre lo penal y lo psiquiátrico}

Un aspecto significativo en el caso de Luis es el persistente auxilio de la psiquiatría - a través de informes y pericias varias- para fundamentar diferentes decisiones judiciales, aun cuando los informes técnicos no son vinculantes. Este rol de la psiquiatría como auxiliar del derecho penal fue acertadamente descrito por Foucault (2000) como una condición de posibilidad de esta para erigirse en su forma moderna, es decir, como especialidad médica.

Así, la tarea de la psiquiatría consistió en marcar el carácter de peligroso del loco y establecer una interrelación entre locura y crimen. Para Foucault, esta capacidad le permitió a la psiquiatría erigirse como disciplina capaz de reconocer y prever la comisión de crímenes relacionados con la locura.

El efecto de esto fue el acoplamiento entre los problemas del sistema penal para ejercer el castigo y las exigencias de la psiquiatría para establecerse como disciplina relevante (Foucault, 2000). Aunque desde entonces la psiquiatría ha experimentado profundos cambios, este resorte (que le permitió erigirse como disciplina capaz de demostrar que en el fondo de cualquier locura está latente un crimen) continúa operando de manera inobjetable, y las pericias psiquiátricas exponen su vigencia en el caso de Luis. El auxilio de la psiquiatría apacigua a lo que elocuentemente Foucault denominó la angustia de juzgar (Foucault, 1991, p. 125).

Volviendo a Luis, el análisis del expediente arroja multiplicidad de informes y pericias que asignan diagnósticos con lógicas clasificatorias no unificadas en torno a un encuadre teórico homogéneo; dichos informes derivan en la prescripción de medicación psicoactiva, con distintas acciones terapéuticas. El primer informe médico que consta en el expediente fue elaborado en una comunidad terapéutica; consigna un diagnóstico que conjuga elementos psicoanalíticos con otros de la biopsiquiatría, teniendo como su foco el consumo de sustancias.

25/01/1996. Motivo de consulta: drogodependencia con consecuencias de conductas antisociales. «Estructura neurótica de personalidad, buena adaptación, aún persiste el reclamo de traslado; conciencia de situación y no de enfermedad».

Se detalla la medicación suministrada, por nombre comercial y dosis. Sintéticamente, se trata de antialucinógenos, ansiolíticos, antipsicóticos y neurolépticos que suelen indicarse para tratar problemas de conducta, esquizofrenia y bipolaridad. El dictamen del informe 
añade otros diagnósticos, puntualmente trastornos adaptativos con trastornos de comportamiento, y destaca el abuso de cocaína.

Dos semanas después, y tras un fallido intento de fuga que incluyó amenazas y agresiones a una enfermera, la lectura cambia y el foco queda en la agresividad, que fundamenta la necesidad de traslado de Luis a una clínica psiquiátrica. En palabras del director de la comunidad terapéutica:

7/02/1996: «Porque nuestra institución es una comunidad terapéutica de tipo tradicional y no disponemos de pisos o celdas de aislamiento de agresividad descontrolada». (Informe del director)

A un mes del primer informe, el cambio de foco es patente. El segundo informe señala «personalidad de base neurótica violencia latente, auto y heteroagresivadad, revistiendo el cuadro características de alta peligrosidad». Se indica otro conjunto de medicamentos psicoactivos. Sucesivos informes acentúan diversas cuestiones, entre ellas:

«Problemas de apoyo familiar.»

«Problemas de toda índole.»

«Politoxicofilia en adolescencia, con alta tendencia al acting out y acting in.»

«Trastorno desadaptativo reiterativo persistente, que produce un período de atención breve, haciendo imposible cortar el acto.»

«Pronóstico reservado. Amenaza constantemente con agredirse.»

«Demuestra ser incontrolable o incorregible.»

«Paciente muy peligroso, auto y heteroagresivo.»

«Paciente obnubilado por el efecto medicamentoso, pero mantiene una actitud querellante de agresividad contenida negándose en la entrevista a brindar datos personales.»

«Paciente de aspecto descuidado, desaseado.»

«Consume desde los 8 años de todo.»

Un registro posterior resume:

14/05/1997: «no es alienado, no es demente en sentido jurídico. Reviste la forma clínica de trastornos graves de la peligrosidad con componentes toxicofílicos y conductas marginales riesgosas» a lo que «se agregan las lesiones descritas por autolesión».

De las resonancias analíticas que admite la lectura de estos fragmentos, señalamos un contrapunto conceptual que interesa profundizar cuando se trata de adolescentes y 
jóvenes: que Luis no era punible cuando fueron realizados estos registros. Se trata del contrapunto entre peligrosidad y riesgo, como dos matrices de inteligibilidad para analizar los discursos que tematizan a adolescentes y jóvenes en relación a la responsabilidad penal en Argentina (Guemureman \& Bianchi, 2019).

En la psiquiatría legal clásica del siglo XIX la peligrosidad se asociaba a la posibilidad de comisión de actos imprevisibles y violentos, al acecho en el interior del individuo, y que contenía la latencia de una conflictividad externa (Foucault, 1990). Para las décadas de 1970 y 1980, la peligrosidad describió un giro que significó una mutación en la terminología, que pasó a considerarse en relación a factores, situaciones y probabilidades estadísticas, reconfigurándose conceptualmente como riesgo. Esto supone una mutación sutil pero significativa en el modo de entender los problemas de salud mental.

Aunque la noción de riesgo y su utilización como parámetro en el diagnóstico en salud mental contiene múltiples aristas (Bianchi, 2012, 2015), destacamos que la configuración del diagnóstico psiquiátrico en la matriz del riesgo no se limita a aspectos vinculados a la salud mental en lo inmediato, sino que incluye además otros tópicos; en ellos se cuentan los antecedentes de empleos, la convivencia familiar, la administración del dinero, la capacidad de compra de bienes, la información sobre las conductas efectuadas en el pasado y el comportamiento peligroso (Rose, 1998).

Estas matrices conceptuales no designan épocas históricas reemplazables o sucesivas, sino capas de sentidos y lógicas argumentales que coexisten de modos complejos. En el caso puntual de Luis, la peligrosidad y el riesgo tributan a la configuración de los numerosos diagnósticos que se superponen, reemplazan, acumulan o desplazan en el expediente. Estos justifican las sistemáticas intervenciones psicofarmacológicas y refuerzan lo que Rose (1996) entiende como prácticas divisorias que separan el yo prudente del yo imprudente; es decir, que dividen a los yo que demuestran ser capaces de gestionarse a sí mismos, de aquellos yo que, en virtud de no demostrar esa capacidad de autogestión, deben ceder su autoadministración y pasar a ser administrados por otros.

Estos diagnósticos, además, son esgrimidos como motivos (tanto de médicos y psiquiatras, como de las autoridades de instituciones que alojaron a Luis) para exigir traslados, y fundamentan los numerosos episodios de fugas registrados en el expediente. A este vector dedicamos el siguiente apartado. 


\section{Trayectorias cíclicas: el tránsito y retorno entre}

\section{instituciones}

Luis contaba con varios legajos tutelares abiertos simultáneamente, en los que se registraban aspectos vinculados a la administración de su vida, informes de delegados tutelares y equipos profesionales de las instituciones, entre otros. En cada juzgado interviniente, respecto de alguna acción de Luis, se abría un nuevo legajo tutelar, a veces sin tenerse conocimiento de que ya estaba abierto otro legajo en otro juzgado. Cuando el juzgado que había abierto el último legajo se anoticiaba de la existencia de otros en curso en otros juzgados, las actuaciones se remitían recíprocamente.

Esta simultaneidad de legajos abiertos dota al expediente de Luis de características peculiares. Entre otras cuestiones, ya que a la dificultad de secuenciar las intervenciones registradas se suma la superposición de intervenciones y oficios librados. En la medida que Luis transitaba por distintos juzgados, se configuraban los trazos de una imagen que años después ya ofrecería una forma consolidada: la un joven desinteresado por transformar su actitud irreductible, violenta e indolente.

Desde la primera aprehensión, el rutero institucional de Luis describe alternancias por tipo y tiempos, en las que se suceden instituciones penales, comunidades terapéuticas y clínicas psiquiátricas. Una trayectoria institucional reiterada a lo largo del expediente consistía en que Luis era aprehendido por la policía y enviado al juzgado, en donde el juez a cargo sabía que tendrían problemas para hospedarlo en alguna institución, debido a sus trayectorias previas. Aquí se abrían diferentes posibilidades. Si se lo enviaba a una institución de seguridad, la misma aducía que no era adecuada a su problemática, porque contenía una base adictiva fuerte. Si se lo remitía a una comunidad terapéutica de régimen semiabierto, desde esta se insistía que no era apto para el tratamiento, dado que no exhibía voluntad de cooperar. Si se lo mandaba a una comunidad terapéutica cerrada, de tipo dual, que alternaba prestaciones con las de clínica psiquiátrica, la queja era que en ausencia de medicación psicoactiva el adolescente incrementaba su violencia, provocando disturbios y agresiones a los demás o a sí mismo. Lo anterior se presente de manera esquemática en la figura 1.

En estos ruteros, las fugas se reiteran y se acompañan de mecanismos de expulsión que operan con mayor o menor sistematicidad. 
Figura 1

Esquema de las trayectorias institucionales de Luis

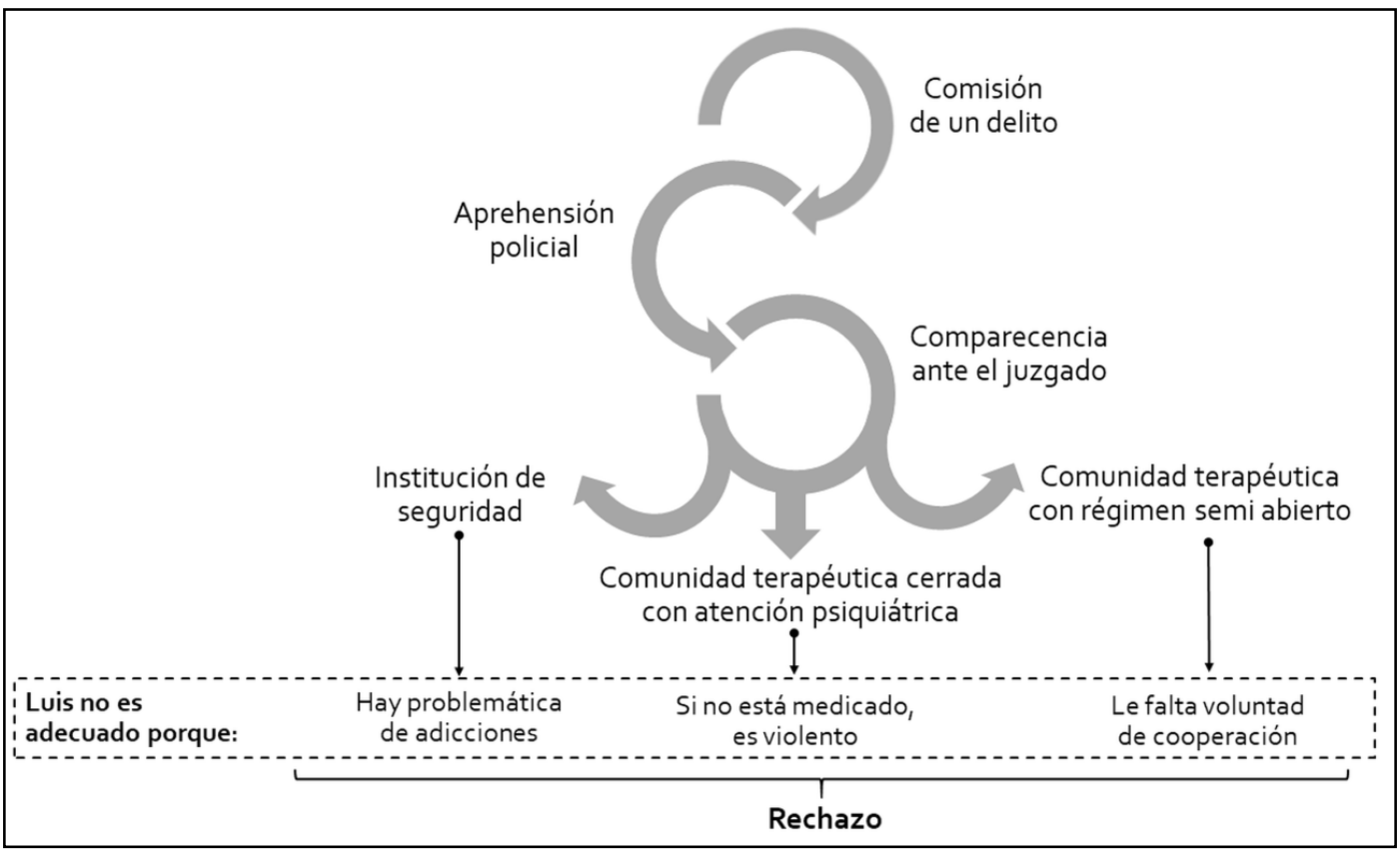

Un hallazgo de la investigación de Guemureman y Daroqui (2001) en juzgados de menores de la provincia de Buenos Aires en el período 1992-1999 les permitió concluir que

las fugas se producen mayoritariamente de las comunidades terapéuticas, y son protagonizadas en forma preponderante por los jóvenes derivados por causas penales, los mismos que una vez en el juzgado, solicitaron ser remitidos a una comunidad para «recuperarse» de sus adicciones, su «petición« fue concedida y, una vez allí, habiendo logrado su objetivo, responden fugándose. (p. 194)

Adicionalmente, sostienen que, en virtud de los testimonios recogidos en sus estudios, las demandas de adolescentes y jóvenes que infringen la ley penal no suelen tenerse en cuenta, salvo cuando se hace patente la expresión de un supuesto deseo de recuperación, redención o expiación. Frente a esta demanda, la internación en comunidades terapéuticas es considerada antes como cura, que como castigo. Como una continuidad con esta consideración de la derivación a la comunidad terapéutica, la respuesta de los adolescentes se explica como un escape. Esta lectura, en la que - en cualquiera de los casosel desenlace esperado es la fuga, omite los mecanismos de expulsión en cada una de esas instancias, que el rutero institucional de Luis pone en evidencia ostensiblemente. 
Así, la trayectoria institucional de Luis es expresiva de un proceso más amplio, de acuerdo con el que

si bien las internaciones en su mayoría no se originan en derivaciones penales, esta suerte de psiquiatrización de problemáticas sociales, inscribe en las instituciones psiquiátricas población otrora institucionalizada en lo instituciones de atención integral, hogares convivenciales y demás instancias que dejaban en claro el carácter no penalizador de la restricción de libertad. (Guemureman, 2012, p. 81)

Por ello, las internaciones psiquiátricas y derivaciones a comunidades terapéuticas podrían operar como un escape a las internaciones tradicionales de índole penal en institutos de seguridad.

Vale aclarar que estas trayectorias cíclicas de ruteros institucionales acontecieron en un período previo a la sanción de la Ley Nacional de Protección de la Salud Mental, 26657 (Argentina, 2010). Esta hubiera dado a Luis el estatuto de padeciente, en virtud de sus problemáticas de consumo de sustancias y de las múltiples clasificaciones de corte psiquiátrico que establecieron los profesionales tratantes.

En el expediente de Luis se registraron numerosos episodios bajo la denominación de fuga y en menor cantidad bajo la categorización de traslados en instituciones que se calificaban como clínicas, institutos de menores o centros de cuidados. Como las secuencias de los diferentes episodios se solapan, no es posible realizar una operación de suma simple. Antes bien, vale traer los matices de los registros al respecto, con menciones a:

- una fuga fallida para Luis, pero exitosa para su compinche;

- la responsabilidad por la organización de un motín con intención de fuga, también fallida;

- la promesa de no fugarse, incumplida a menos de una semana de pronunciada;

- traslados reversibles entre un instituto y un centro de cuidados;

- fuga de una clínica, seguida de aprehensión, y nueva fuga a la semana;

- traslado a una clínica «elegida por contar con dispositivos para evitar fugas a las que es afecto el causante» (nota del juzgado);

- «alto riesgo con respecto a las fugas» (planilla de control semanal de la clínica).

En todos los casos, la internación da lugar a un pedido institucional de traslado a otras instituciones, más específicas, donde lo que prima es alejar a Luis; premisa a cuya 
consecución el saber psiquiátrico contribuye para proveer fundamentos y ofrecer terapéuticas, generalmente por medio de medicamentos. Como ejemplo, extractamos un registro del expediente:

19/10/1996: Se avisa que el menor se fuga luego de agredir a una enfermera, y se realizó una denuncia por la agresión y un aviso de la fuga. (Oficio de la clínica)

Hasta el día anterior, la respuesta al tratamiento venía siendo buena (consta un detalle de prescripción de psicofármacos, antialucinógenos, neurolépticos, ansiolíticos y un protector gástrico). Pero ese 20 de octubre era el Día de la Madre y Luis había manifestado su voluntad de visitar a la suya. Sin embargo, no tenía permiso para hacerlo; ante sus reclamos, le aumentaron la medicación habitual. Como resultado, según consta en el expediente y de acuerdo a las palabras de una enfermera «no podía tenerse en pie solo», pero ayudado por dos compañeros, el 19 de octubre se fugó.

Una síntesis de este vector la da el director de una clínica en la que Luis estuvo internado, quien a fines de 1996 expone el caso en el juzgado interviniente, basando su solicitud de traslado en que Luis es un «paciente que no se beneficia de los cuidados psiquiátricos, sino que los aprovecha para fugarse», con acciones en las que la violencia aparece cada vez más frecuentemente. Y las violencias en su doble faz de padecimiento y ejercicio son, precisamente, el tercer nodo de trayectorias a tratar.

\section{Trayectorias permanentes y crecientes de violencias asociadas}

Las trayectorias analizadas en las dos secciones anteriores convergen en la configuración del perfil de Luis como un irreductible, desidioso y refractario a acciones de mejora y con problemas psiquiátricos de creciente severidad. Estas caracterizaciones dispersas se unifican en torno a la antedicha peligrosidad, y tienen su epicentro de justificación en el registro de una sucesión de hechos asociados a problemas de violencia.

De modo que el tercer vector se relaciona con las persistentes, aunque in crescendo, trayectorias de violencias padecidas y ejercidas registradas en el expediente. En las páginas que lo conforman, las violencias múltiples y combinadas dibujan un hilo conductor que cohesiona las trayectorias híbridas entre lo psiquiátrico y lo penal, así como las trayectorias institucionales, cíclicas y enmarañadas. 
La sucesión de internaciones, fugas, aprehensiones, nuevas fugas y traslados están siempre mediadas por la irrupción de diferentes violencias. Esta tónica, sin embargo, se inició antes del primer contacto con el juzgado, ya que el expediente traza una historia familiar de abandono, malos tratos y falta de contención. El modo que encuentra Luis para vincularse con estos sucesos de su vida es reactivo: trata mal y agrede a quienes lo tratan bien. Mayormente prefiere el silencio. Si habla, es a través de sus actos que siempre incluyen el ejercicio de la violencia. Conoce el lenguaje de la violencia y la ley de la fuerza; por eso, redobla las apuestas con sus acciones. Se comunica a través de la violencia y, cuando usa la palabra, es para solicitar que no le hablen, «que no le hagan la cabeza», «que no intenten convencerlo»y, lo más notorio, «que no lo traten bien, que no está acostumbrado, que siempre lo trataron a los golpes».

Foucault analizó cómo el poder disciplinario detecta y expone irreductibles, inclasificables, mientras que simultáneamente crea nuevos dispositivos por el accionar de los cuales se hace un intento por restablecer la regla. Y esto es así porque «los sistemas disciplinarios se caracterizan por un trabajo constante de la norma en la anomia» (Foucault, 2005, p. 76), identificando los residuos del poder disciplinario y creando instituciones cada vez más específicas en las que inscribirlos para su reinserción. En esta línea, Luis encarna el residuo de los residuos, el institucionalmente inclasificable por excelencia, permanentemente excluido, incluso de los lugares de exclusión. Algunos registros en función del análisis del expediente:

7/02/1996: Se reporta que Luis y otro joven han tomado del cuello a la enfermera de guardia, han ingresado a la enfermería y, tomando una tijera, han amenazado a la misma con dañarla si no abre la puerta. Le han robado las llaves y la han amenazado de muerte. La fuga es exitosa para el otro joven, pero no para Luis. Sin embargo, desde la clínica piden urgente el traslado a una clínica psiquiátrica. El fundamento del pedido: «Porque nuestra institución es una comunidad terapéutica de tipo tradicional y no disponemos de pisos o celdas de aislamiento de agresividad descontrolada». (Informe del director de clínica)

Mediados de 1997: se continúan buscando alternativas en organismos no gubernamentales, a través del Organismo Técnico Administrativo (entonces, el Consejo del Menor y la Familia), que eleva informes al Juzgado explicando la situación y los motivos por los que ninguna institución quiere aceptar al paciente, que «no acepta pautas de tratamiento ni convivencia alguna», y se replican los informes sobre los episodios de violencia. Distintos centros de cuidados y clínicas refieren episodios anteriores de violencia y agresión. 
A medida que los registros del expediente avanzan en el tiempo, se acelera la secuencia, hasta que Luis es derivado a unidades penitenciarias. Para cuando cumple la mayoría de edad, a los ruteros de fugas, traslados, diagnósticos, psicofármacos, sanciones y violencias se anexan informes de conducta y concepto elaborados por las unidades penitenciarias, que lo trasladan cada vez que su conflictividad excede lo tolerable.

La segunda sentencia dictamina una condena sin reducción de diez años de prisión, producto de las causas tramitadas ante ese tribunal, así como la unificación con alguna sentencia condenatoria, ya dictada por un tribunal ordinario.

Esta condena pone en escena el fracaso del tratamiento tutelar. La expedición taxativa y unánime del tribunal se sustenta en la «pésima impresión causada por el enjuiciado» en la audiencia de conocimiento personal prevista en el artículo $41^{\underline{0}}$ del Código Penal, Ley 11179 (Argentina, 1984), que no cumplió las pautas, no modificó su conducta, no se esforzó por seguir las recomendaciones profesionales y no discontinuó la comisión de acciones antisociales, tanto intra como extramuros. Su declaración es elocuente: «Me gusta consumir, me gusta robar», y ello tributa a la configuración del perfil antedicho.

Considerando que los hechos objeto de reproche penal consistieron, mayoritariamente, en robos simples, la pena aplicada es extremadamente elevada. Aunque el monto puede ser aplicado en función de la cantidad de hechos, es destacable mencionar lo inhabitual de que, tratándose de un menor de edad, se haya aplicado una condena tal, especificando que se accedía a la pretensión fiscal. El dictado de una condena sin reducción marca el fin de los registros a los que se accedió en el relevamiento del expediente y segunda sentencia de Luis.

\section{Discusión}

La constatación general que surge del análisis es que, como formulamos en la hipótesis, el caso de Luis describe trayectorias de exclusión combinadas, densamente relacionadas entre sí. La división en tres ejes arrojó que uno de los vectores se relaciona con los diagnósticos psiquiátricos asociados a los ruteros institucionales de Luis, que expresan entre otras cuestiones, el auxilio y la hibridación del saber psiquiátrico en relación a la fundamentación de las decisiones judiciales. Estos diagnósticos conjugan elementos de corte netamente clínico-orgánicos, con consideraciones de tipo vincular y comportamental. Este entramado de diagnósticos y su correlativa terapéutica basada en medicamentos 
ofician como pilares de fundamentación para los numerosos episodios de fuga y exclusiones recurrentes de las diferentes instituciones por las que Luis fue pasando de acuerdo a las constancias del expediente.

El segundo vector apunta a la ciclicidad y sinuosidad de las trayectorias institucionales por las que transitó Luis. Documentamos diferentes instituciones por las que él circuló y establecimos distintos mecanismos que generaron expulsiones de cada una de ellas. Una regularidad en las fundamentaciones de los rechazos institucionales aparece en torno a las acciones violentas, ejercidas por Luis en estos ruteros.

El tercer vector ahonda en estas violencias, que se combinan con las que Luis ha padecido tanto previamente como durante sus institucionalizaciones. Ambas violencias tienen carácter de permanencia y van in crescendo a lo largo de los registros. La recurrencia y profundización de las trayectorias de violencias concurren con los demás vectores en la aplicación de una pena extremadamente alta y unánime. Entonces, los tres vectores de trayectorias no establecen caminos lineales, pero sí convergentes en relación a un desenlace que deviene en la remisión en el sistema penal.

El análisis también evidencia la falta de articulación entre el sistema de protección y el sistema de responsabilidad penal en lo que refiere a jóvenes y adolescentes. En esta línea, casos como el analizado muestran que es imperativo, por una parte, atender a que las intervenciones sobre la niñez vulnerable deben ser integrales desde muy temprana edad. Por otra parte, expone que es necesario reforzar sistemas de protección en todos los eslabones, abarcando tanto la atención de salud, como los problemas de consumos problemáticos de sustancias, problemáticas de hábitat y la promoción de las familias.

El caso de Luis también transparenta, como en otros casos de adolescentes que proceden de familias sobrevulneradas, el vacío que se produce en los derechos y las insuficientes respuestas público-estatales. La no intervención en estas claves expele a los niños de la órbita de la protección y de las dinámicas familiares, arrojándolos a la condición de residuos que, a fuerza de desatención, cronifican su situación y permanecen en una suerte de limbo institucional en el que el rechazo y la fuga se integran como dos modos reversibles de circulación por instituciones jurídicas y psiquiátricas, hasta que reúnen las condiciones para ser recibidos por el sistema penal. 


\section{Referencias}

Agüero, C. (2014) ¿Conforman las sentencias penales un género discursivo? Estudios Filológicos, (53), 7-26. https://doi.org/10.4067/s0071-17132014000100001

Agüero, C., \& Zambrano, J. P. (2009). La narración en las sentencias penales. Universum, 24(2), 28-41. https://doi.org/10.4067/so718-23762009000200003

Archenti, N. (2007). Estudio de caso/s. En A. Marradi, N. Archenti, \& J. I. Piovani

(Comps.), Metodología de las ciencias sociales (pp. 237-246). Emecé.

Argentina. (1980). Decreto Ley 22 278. Régimen Penal de la Minoridad. https://bit.ly/2CXJKgu Argentina. (1984). Ley 11179. Código Penal de la nación argentina. Infoleg.

Argentina. (2006). Resolución 2857 del ex Ministerio de Educación, Ciencia y Tecnología.

Lineamientos para el comportamiento ético en las Ciencias Sociales y Humanidades, Comité de Ética de Conicet. Consejo Nacional de Investigaciones Científicas y Técnicas.

Argentina. (2010). Ley nacional 26 657. Derecho a la protección de la salud mental. Infoleg. https://bit.ly/3gtZDJd

Bajtin, M. (1982). Estética de la creación verbal. Siglo XXI.

Becker, H. (1974). Los extraños. Tiempo Contemporáneo.

Beloff, M. A. (2004). Un modelo para armar iy otro para desarmar! Protección integral de derechos del niño vs. derechos en situación irregular. En M. A. Beloff (Ed.), Los derechos del niño en el sistema interamericano (pp. 1-45). Ediciones Del Puerto.

Beloff, M. A., \& García-Méndez, E. (2004). Infancia, ley y democracia en América Latina. Temis.

Bianchi, E. (2012). El problema del riesgo: notas para una reflexión sobre los aportes surgidos de la usina genealógica en torno al concepto de riesgo en salud mental. Espacios Nueva Serie, 1(7), 84-97.

Bianchi, E. (2015). «El futuro llegó hace rato»: susceptibilidad, riesgo y peligrosidad en el diagnóstico y tratamiento por TDAH en la infancia. Revista de Estudios Sociales, (52), 185-199. https://doi.org/10.7440/res52.2015.13

Bourdieu, P. (200o). La fuerza del derecho: elementos para una sociología del campo jurídico. Palimpsesto.

Bourdieu, P., \& Passerón, J.-C. (1996). La reproducción: elementos para una teoría del sistema de enseñanza. Laia.

Chávez, M. (2010). Jóvenes, territorios y complicidades: una antropología de la juventud urbana. Espacio. 
Chávez, M., \& Fidalgo-Zeballos, E. (2013). Políticas de infancia y juventud: producir sujetos y construir estado. Espacio.

Cloward, R., \& Ohlin, L. (2000). Delinquency and opportunity: A theory of delinquent gangs. Routledge. https://doi.org/10.4324/9781315007274

Cohen, A. (1955). Delinquent boys: The culture of the gangs. Free Press.

Cucatto, M. (2009). La conexión en las sentencias penales de primera instancia: del análisis de textos a la práctica de escritura de sentencias. Revista de Llengua i Dret, (51), 135-160.

Dávila-León, O. \& Guiardo-Soto, F. (2005). Trayectorias, transiciones y condiciones juveniles en Chile. Revista Nueva Sociedad, (200), 114-126.

Ferrarotti, F. (1988). Biografía y ciencias sociales. Flacso.

Ferrarotti, F. (1991). La historia y lo cotidiano. Península.

Flyvbjerg, B. (2006). Cinco malentendidos acerca de la investigación mediante los estudios de caso. Reis, (106), 33-62. https://doi.org/10.2307/40184584

Foucault, M. (1990). La vida de los hombres infames. La Piqueta.

Foucault, M. (1991). La angustia de juzgar: debate sobre la pena de muerte. En J. Varela \& F. Álvarez-Uría (Eds.), Saber y verdad (pp. 107-126). La Piqueta.

Foucault, M. (2000). Los anormales. Fondo de Cultura Económica.

Foucault, M. (2005). El poder psiquiátrico. Fondo de Cultura Económica.

García-Méndez, E., \& Bianchi, M. del C. (1991). Ser niño en América Latina: de las necesidades a los derechos. Galerna.

García-Méndez, E., \& Carranza, E. (1992). Del revés al derecho: la condición jurídica de la infancia en América Latina. Bases para una reforma legislativa. Galerna.

Guemureman, S. (2011). La cartografía moral de las prácticas judiciales en los Tribunales de Menores: los Tribunales Orales en la Ciudad de Buenos Aires. Ediciones del Puerto.

Guemureman, S. (2012). Aproximaciones a la realidad del encierro de adolescentes y jóvenes en la Argentina. En A. Daroqui, S. Guemureman, C. Motto, N. Maggio, J. Pasin, N. Debandi, M. Bouilly, A. L. López, J. Andersen, \& H. Motta (Orgs.), Seminario de estudios comparados sobre las estrategias del gobierno de la cárcel neoliberal en Argentina y en Francia (pp. 54-87). Universidad de Buenos Aires; Instituto de Investigaciones Gino Germani; Grupo de Estudios sobre Sistema Penal y Derechos Humanos.

Guemureman, S. (2018). El tríptico el secreto, el silencio y la exclusión en las tramas de violencia y padecimiento psíquico de niños. Psicoperspectivas. Individuo y Sociedad, ${ }_{17}(2), 1$-11. https://doi.org/10.5027/psicoperspectivas-vol17-issue2-fulltext-1217 
Guemureman, S., \& Bianchi, E. (2019). Riesgos no tan explícitos y peligros no tan solapados: un análisis de los proyectos de ley sobre responsabilidad penal juvenil en Argentina 2016-2018. Revista Derecho y Ciencias Sociales, (20), 1-22. https://doi.org/ $10.24215 / 185229710045$

Guemureman, S., \& Daroqui, A. (2001). La niñez ajusticiada. Ediciones del Puerto.

Kossoy, A. (2012, 24 y 25 de abril). Trayectorias subjetivas y construcción de identidad social de jóvenes de clases populares [Ponencia]. VII Jornadas de Sociología, Buenos Aires, Argentina.

Lemert, E. M. (1967). Estructura social, control social y desviación. En M. B. Clinard (Comp.), Anomia y conducta desviada (pp. 64-100). Paidós.

Macri, M., \& Uhart, C. (2017). Niñas, niños y adolescentes trabajadores: trayectorias socioeducativas y laborales. Biblos.

Mallimaci. F., \& Giménez-Béliveau, V. (2006). Historia de vida y métodos biográficos. En I. Vasilachis de Gialdino (Coord.), Estrategias de investigación cualitativa (175-212). Gedisa.

Mari, E. (2002). La teoría de las ficciones. Eudeba.

Martínez-García, J. I. (1992). La imaginación jurídica. Debate.

Matza, D. (1981). El proceso de desviación. Taurus.

Neiman, G., \& Quaranta, G. (2006). Los estudios de caso en la investigación sociológica. En I. Vasilachis de Gialdino (Coord.), Estrategias de investigación cualitativa (213-238). Gedisa.

Pardo, M. L. (1992). Derecho y lingüistica: cómo se juzga con palabras. Análisis lingüistico de sentencias judiciales. Centro Editor de América Latina.

Parodi, M. L. (2008). Géneros del discurso escrito: hacia una concepción integral desde una perspectiva sociocognitiva. En G. Parodi (coord.), Géneros académicos y géneros profesionales: accesos discursivos para saber y hacer (17-39). Ediciones Universitarias de Valparaíso.

Parodi, G. (2009). Géneros discursivos y lengua escrita: propuesta de una concepción integral desde una perspectiva sociocognitiva. Letras, 51(80), pp. 19-56.

Reiter, H. (2003). Past, present, future. Biographical time structuring of disadvantaged young people. Young, 11(3), 253-279. https://doi.org/10.1177/11033088030113004

Rose, N. (1996). Psychiatry as a political science: Advanced liberalism and the administration of risk. History of the Human Sciences, 9(2), 1-23. https://doi.org/ $10.1177 / 095269519600900201$ 
Rose, N. (1998). Governing risky individuals: The role of psychiatry in new regimes of control. Psychiatry, Psychology and Law, $5(2), 177-195$. https://doi.org/ 10.1080/13218719809524933

Rovacio, A., Galetto, S., Arias, L., \& Pacheco, A. (2016). Estudios sobre juventudes en Argentina IV: Juventudes. Campo de saberes y campo de intervención. De los avances a la agenda aún pendiente. Nueva Editorial Universitaria.

Stake, R. (1994). Case studies. En N. K. Denzin \& Y. S. Lincoln (Eds.), Handbook of qualitative research (236-247). Sage.

Torillo, D. (2011). Las trayectorias laborales y sociales de mujeres de sectores populares beneficiarias del Plan Familias: un estudio de caso en un barrio de la periferia sur de la Ciudad de La Plata [Tesis de Maestría no publicada]. Universidad de Buenos Aires.

Van Dijk, T. (2003). La multidisciplinaridad del análisis crítico del discurso: un alegato en favor de la diversidad. Métodos de análisis crítico del discurso. Gedisa.

Vasilachis de Gialdino, I. (1992). Métodos cualitativos I: los problemas teórico-epistemológicos. Centro Editor de América Latina.

Yin, R. (2014). Case study research: Design and methods. Sage. 